\title{
Autofocusing in optical scanning holography
}

\author{
Taegeun $\mathrm{Kim}^{1, *}$ and Ting-Chung Poon ${ }^{2}$ \\ 'Department of Optical Engineering, Sejong University, 98 Kunja-dong, \\ Kwangjin-gu, Seoul 134-747, South Korea \\ ${ }^{2}$ Department of Electrical and Computer Engineering, Virginia Tech, Blacksburg, Virginia 24061, USA \\ ${ }^{*}$ Corresponding author: takim@sejong.ac.kr
}

Received 13 July 2009; revised 12 October 2009; accepted 12 October 2009; posted 12 October 2009 (Doc. ID 114193); published 3 November 2009

\begin{abstract}
We present autofocusing in optical scanning holography $(\mathrm{OSH})$ with experimental results. We first record the complex hologram of an object using OSH and then create the Fresnel zone plate (FZP) that codes the object constant within the depth range of the object using Gaussian low-pass filtering. We subsequently synthesize a real-only spectrum hologram in which its phase term contains information about a distance parameter. Finally, we extract the distance parameter from the real-only spectrum hologram using fringe-adjusted filtering and the Wigner distribution. Using the extracted distance parameter, we reconstruct a three-dimensional image of the object from the complex hologram using digital convolution, which bypasses the conventional blind convolution to reconstruct a hologram. To the best of our knowledge, this is the first report with experimental results that autofocusing in OSH is possible without any searching algorithm or tracking process. (C) 2009 Optical Society of America

OCIS codes: $\quad 090.1995,070.0070$.
\end{abstract}

\section{Introduction}

Three-dimensional (3-D) imaging using optical scanning holography (OSH) has a long-standing history [1-4]. Most recently, OSH has been applied to 3-D microscopy [3-6], and an achieved resolution of better than $1 \mu \mathrm{m}$ has been reported [5]. OSH is a form of digital holography [7] but it records a complex hologram by two-dimensional heterodyne scanning. Identical to conventional reconstruction of digital holograms, sectioning reconstruction of a 3-D image is done by convolving a complex conjugate of the Fresnel zone plate (FZP) matched to the depth of a section of a 3-D object [7]. Since we do not have prior knowledge of the depth location of the object, we need to perform digital reconstruction blindly to various distances until we find the sectional images, which is a time-consuming process. Several numerical techniques have been proposed to extract the distance parameter automatically [8], but these involve a search algorithm $[9,10]$ or tracking process [8]. Here

0003-6935/09/34H153-07\$15.00/0

(C) 2009 Optical Society of America we show experimentally that reconstruction of the focused image sections from $\mathrm{OSH}$ is possible without any searching algorithm or tracking process. First, we record the complex hologram of an object by use of OSH. Second, we perform Gaussian low-pass filtering with a complex hologram, which makes the FZP that codes the object constant within the depth range of the object [11]. Third, we synthesize the real-only spectrum hologram from the Gaussian low-pass filtered hologram. We then show that, since the FZP that codes the object is constant within the depth range of the object and the intensity of the object is positive and real, the phase term of the realonly spectrum hologram contains information about the distance parameter. Fourth, we separate the phase term of the real-only spectrum hologram using fringe-adjusted filtering [12]. Fifth, we extract the distance parameter by performing the Wigner distribution of the phase term of the real-only spectrum hologram [13]. Finally, we reconstruct the entire 3-D image of the object by reconstruction using a distance parameter, which avoids the blind convolution normally used for digital reconstruction. 


\section{Complex Hologram Recording and Distance Parameter Extraction}

\section{A. Recording with Optical Scanning Holography}

In the recording stage, we record a complex hologram of an object using OSH. The OSH setup shown in Fig. 1 consists of a Mach-Zehnder interferometer and an electronic processing unit. Two mirrors (M2 and M3) and two beam splitters (BS1 and BS2) form the Mach-Zehnder interferometer. The laser beam generated by the He-Ne laser is split into two paths. The frequencies of the laser in the upper path and the lower path beams are shifted by $\Omega$ and $\Omega+\Delta \Omega$, respectively, through acousto-optic frequency shifters (AOFS1 and AOFS2), and each beam is then collimated by a $10 \times$ beam expander (BE1 and BE2). Afterward, the upper path beam becomes a spherical wave having limited extension through lens L1. The spherical wave and the lower path collimated beam are combined through beam splitter BS2 to form an interference pattern called the time-dependent (TD) FZP with limited extension. The TD FZP at $z$ away from the focal point of lens L1 is given by

$$
I_{s}(x, y, z, t)=\frac{A_{s}(x, y ; z)}{\lambda z} \sin \left[\frac{\pi}{\lambda z}\left(x^{2}+y^{2}\right)-\Delta \Omega t\right],
$$

where $A_{s}(x, y ; z)$ is the size-limiting factor determined by the numerical aperture of the optics that was used to create the TD FZP. In practice, we assume a Gaussian envelope with radius $a(z)$, i.e.,

$$
A_{s}(x, y ; z)=\exp \left[\frac{-\pi}{a(z)^{2}}\left(x^{2}+y^{2}\right)\right] .
$$

Here the radius of the TD FZP is determined by $a(z)=\mathrm{NA} \times z$, where NA represents the numerical

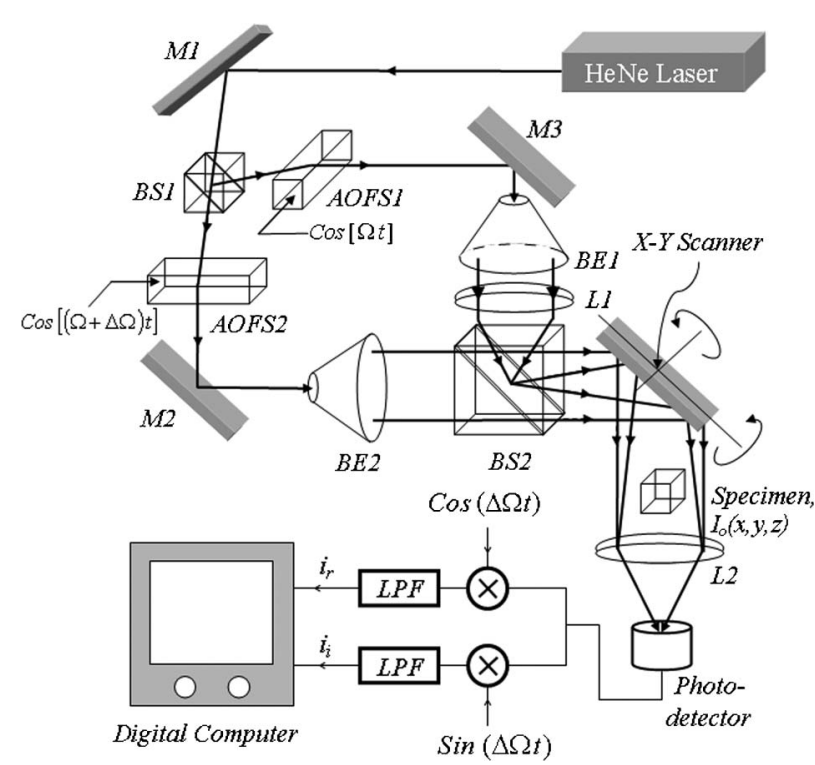

Fig. 1. OSH: M, mirrors; AOFS1, AOFS12, acousto-optic frequency shifters; BS1, BS2, beam splitters; BE1, BE2, beam expanders; L1, focusing lens; L2, collecting lens; $\otimes$, electronic multiplier; LPF, low-pass filter. aperture defined as the sine of the half-cone angle subtended by the TD FZP, which scans an object with intensity transmittance or intensity reflectance, $I_{0}(x, y ; z)$, if the object is diffusely reflecting. Lens $\mathrm{L} 2$ collects all the transmitted light through the object onto the photodetector. Since the lens collects all the transmitted light, the $\mathrm{OSH}$ is in the incoherent mode [4], which means that we record the intensity of the $\overline{3}$-D object holographically. At each scan location, the electric current generated by the photodetector is provided to the electric processing unit shown in Fig. 1. In the electronic processing unit, in-phase and quadrature-phase output currents, $i_{I}(x, y, z)$ and $i_{Q}(x, y, z)$, are generated using a phasesensitive detection scheme such as a lock-in amplifier. Afterward, the in-phase and quadrature-phase output currents are stored in a digital computer according to the scanning locations. The complex hologram is constructed subsequently by adding the two stored outputs in the following manner [14]:

$$
\begin{aligned}
H(x, y)= & i_{I}(x, y, z)-j i_{Q}(x, y, z)=\int_{z_{0}-(1 / 2) \delta z}^{z_{0}+(1 / 2) \delta z} I_{0}(x, y, z) \\
& \otimes \frac{j A_{s}(x, y ; z)}{\lambda z} \exp \left[-j \frac{\pi}{\lambda z}\left(x^{2}+y^{2}\right)\right] \mathrm{d} z,
\end{aligned}
$$

where $z_{0}$ is the distance from the focused point on BS2 that is due to lens L1 to the middle of the object, which we refer to as the distance parameter hereafter, and $\delta z$ is the depth range of the object. The $\otimes$ in Eq. (2) denotes a two-dimensional convolution operation defined as

$$
\begin{aligned}
g_{1}(x, y) & \otimes g_{2}(x, y) \\
& =\iint g_{1}\left(x^{\prime}, y^{\prime}\right) g_{2}\left(x-x^{\prime}, y-y^{\prime}\right) \mathrm{d} x^{\prime} \mathrm{d} y^{\prime} .
\end{aligned}
$$

Note that, in Eq. (2), the object is coded by a Gaussian FZP with a numerical aperture of NA.

The spectrum of the complex hologram is obtained by Fourier transformation of Eq. (2). The Fourier transform operation, $F\{$.$\} , is define \bar{d}$ as

$$
\begin{aligned}
F\{u(x, y)\}_{k_{x}, k_{y}} & =\iint u(x, y) \exp \left[j\left(k_{x} x+k_{y} y\right)\right] \mathrm{d} x \mathrm{~d} y \\
& =\mathbf{u}\left(k_{x}, k_{y}\right),
\end{aligned}
$$

with $\left(k_{x}, k_{y}\right)$ denoting spatial frequencies. Note that the bold letter $\mathbf{u}$ represents the Fourier transform of $u$. Since the spatial frequency of the TD FZP is limited by the NA of the TD FZP [15], the radius of the spectrum of the TD FZP is given by $2 \pi \mathrm{NA} / \lambda$. Thus, the Fourier transform of Eq. (2) is given by 


$$
\begin{aligned}
& \mathbf{H}\left(k_{x}, k_{y}\right)=F\{H(x, y)\}=\int_{z_{0}-(1 / 2) \delta z}^{z_{0}+(1 / 2) \delta z} \mathbf{I}_{0}\left(k_{x}, k_{y}, z\right) \\
& \quad \times \exp \left\{\left[-\frac{1}{4 \pi}\left(\frac{\lambda}{\mathrm{NA}}\right)^{2}+j \frac{\lambda z}{4 \pi}\right]\left(k_{x}^{2}+k_{y}^{2}\right)\right\} \mathrm{d} z .
\end{aligned}
$$

The idea of autofocusing is to extract the value of $z_{0}$, the distance parameter, from Eq. (3) and then to reconstruct the hologram through convolution starting from $z=z_{0}$, thereby bypassing the blind convolution starting at $z \approx 0$ from the hologram.

\section{B. Distance Parameter Extraction}

We first filter the complex hologram using a Gaussian low-pass filter in such a way that the pattern of the FZP that codes the object remains unchanged within the depth range of the object. The Gaussian low-pass filter is given by

$$
\boldsymbol{A}_{g}\left(k_{x}, k_{y}\right)=\exp \left\{-\pi\left[\frac{\lambda}{2 \pi \mathrm{NA}_{g}}\right]^{2}\left(k_{x}^{2}+k_{y}^{2}\right)\right\},
$$

where $\mathrm{NA}_{g}$ determines the cutoff frequency of the filter. In the frequency domain, the Gaussian low-pass filter hologram, $\mathbf{H}_{l p}\left(k_{x}, k_{y}\right)$, is given by multiplying the Gaussian low-pass filter with Eq. (3):

$$
\begin{aligned}
\mathbf{H}_{l p}\left(k_{x}, k_{y}\right)= & \mathbf{H}\left(k_{x}, k_{y}\right) \times \boldsymbol{A}_{\boldsymbol{g}}\left(k_{x}, k_{y}\right) \\
= & \int_{z_{0}-(1 / 2) \delta z}^{z_{0}+(1 / 2) \delta z} \mathbf{I}_{\mathbf{0}}\left(k_{x}, k_{y}, z\right) \\
& \times \exp \left[\left\{-\frac{1}{4 \pi}\left(\frac{\lambda}{\mathrm{NA}_{l p}}\right)^{2}+j \frac{\lambda z}{4 \pi}\right\}\left(k_{x}^{2}+k_{y}^{2}\right)\right] \mathrm{d} z,
\end{aligned}
$$

where $\mathrm{NA}_{l p}=\mathrm{NA}_{g} \mathrm{NA} / \sqrt{\mathrm{NA}^{2}+\mathrm{NA}_{g}^{2}}$. The Gaussian low-pass filtered hologram in the space domain is given by the inverse Fourier transformation of Eq. (5):

$$
\begin{gathered}
H_{l p}(x, y)=F^{-1}\left\{\mathbf{H}_{l p}\left(k_{x}, k_{y}\right)\right\}=\int_{z_{0}-(1 / 2) \delta z}^{z_{0}+(1 / 2) \delta z} I_{0}(x, y, z) \\
\otimes \frac{j A_{l p}(x, y ; z)}{\lambda z} \exp \left[-j \frac{\pi}{\lambda z}\left(x^{2}+y^{2}\right)\right] \mathrm{d} z,
\end{gathered}
$$

where $F^{-1}\{\}$ represents inverse Fourier transformation operation and

$$
A_{l p}(x, y ; z)=\exp \left[\frac{-\pi}{a_{l p}(z)^{2}}\left(x^{2}+y^{2}\right)\right],
$$

with $a_{l p}(z)=\mathrm{NA}_{l p} z$. Note that, in the filtered hologram given by Eq. (6), the object is now coded with a Gaussian FZP with $\mathrm{NA}_{l p}$ instead of the original complex hologram in which the object is coded with a Gaussian FZP with NA. Hence, the Rayleigh range of the Gaussian FZP is now determined by the NA of the FZP, which is given by

$$
\Delta z=2 \lambda / \pi \mathrm{NA}_{l p}^{2}=2 \lambda / \pi\left(\mathrm{NA}^{2}+\mathrm{NA}_{g}^{2}\right) /\left(\mathrm{NA}_{g} \mathrm{NA}\right)^{2} .
$$

When we set $\mathrm{NA}_{g}$ such that the Rayleigh range of the FZP is larger than the depth range of the object, i.e., $\Delta z \geq \delta z$, the radius of the scanning beam pattern is approximately constant within the depth range of the object, i.e., $a_{l p}(z) \approx a_{l p}\left(z_{0}\right)=\mathrm{NA}_{l p} z_{0}$. As a result, the FZP that encodes the complex hologram becomes constant within the depth range of the object, i.e., the free-space impulse response now becomes

$$
\begin{aligned}
h_{z}(x, y) & \approx h_{z_{0}}(x, y) \\
& =j A_{l p}\left(x, y ; z_{0}\right) /\left(\lambda z_{0}\right) \exp \left[-j \frac{\pi}{\lambda z_{0}}\left(x^{2}+y^{2}\right)\right]
\end{aligned}
$$

within the depth range of the object. This is important because we can now pull the factor $h_{z_{0}}(x, y)$ outside the integral of Eq. (6). Under this condition the Gaussian low-pass filtered hologram becomes

$$
\begin{aligned}
H_{l p}(x, y) & =\int_{z_{0}-(1 / 2) \delta z}^{z_{0}+(1 / 2) \delta z} I_{0}(x, y, z) \mathrm{d} z \\
& \otimes \frac{j A_{l p}\left(x, y ; z_{0}\right)}{\lambda z_{0}} \exp \left[-j \frac{\pi}{\lambda z_{0}}\left(x^{2}+y^{2}\right)\right] .
\end{aligned}
$$

Figure 2 is a flow chart. The first two blocks illustrate the procedures discussed so far. After Gaussian lowpass filtering, we synthesize the real-only spectrum hologram to extract distance parameter $z_{0}$. First, we extract the real and imaginary parts of the hologram, which are called the sine hologram and the cosine hologram, respectively. Second, we transform these holograms into the frequency domain, in which we synthesize the real-only spectrum hologram by adding the real parts of the sine hologram and the cosine hologram in the following manner:

$$
\begin{aligned}
\mathbf{H}_{\mathbf{r}-\mathbf{o n l y}}\left(k_{x}, k_{y}\right)= & \operatorname{Re}\left[F\left\{\operatorname{Re}\left[H_{l p}(x, y)\right]\right\}\right] \\
& +j \operatorname{Re}\left[F\left\{\operatorname{Im}\left[H_{l p}(x, y)\right]\right\}\right],
\end{aligned}
$$

which can be shown to become [13]

$$
\begin{gathered}
\mathbf{H}_{\mathbf{r}-\text { only }}\left(k_{x}, k_{y}\right)=\operatorname{Re}\left[F\left\{\int_{z_{0}-(1 / 2) \delta z}^{z_{0}+(1 / 2) \delta z} I_{0}(x, y, z) \mathrm{d} z\right\}\right] \\
\quad \times \exp \left[\left\{-\frac{1}{4 \pi}\left(\frac{\lambda}{\mathrm{NA}_{l p}}\right)^{2}+j \frac{\lambda z_{0}}{4 \pi}\right\}\left(k_{x}^{2}+k_{y}^{2}\right)\right],
\end{gathered}
$$

where $\operatorname{Re}[$.$] and \operatorname{Im}[$.$] are operators that extract$ the real and imaginary parts of a complex number, respectively. Note that, in Eq. (9), the phase term of the real-only spectrum hologram contains 


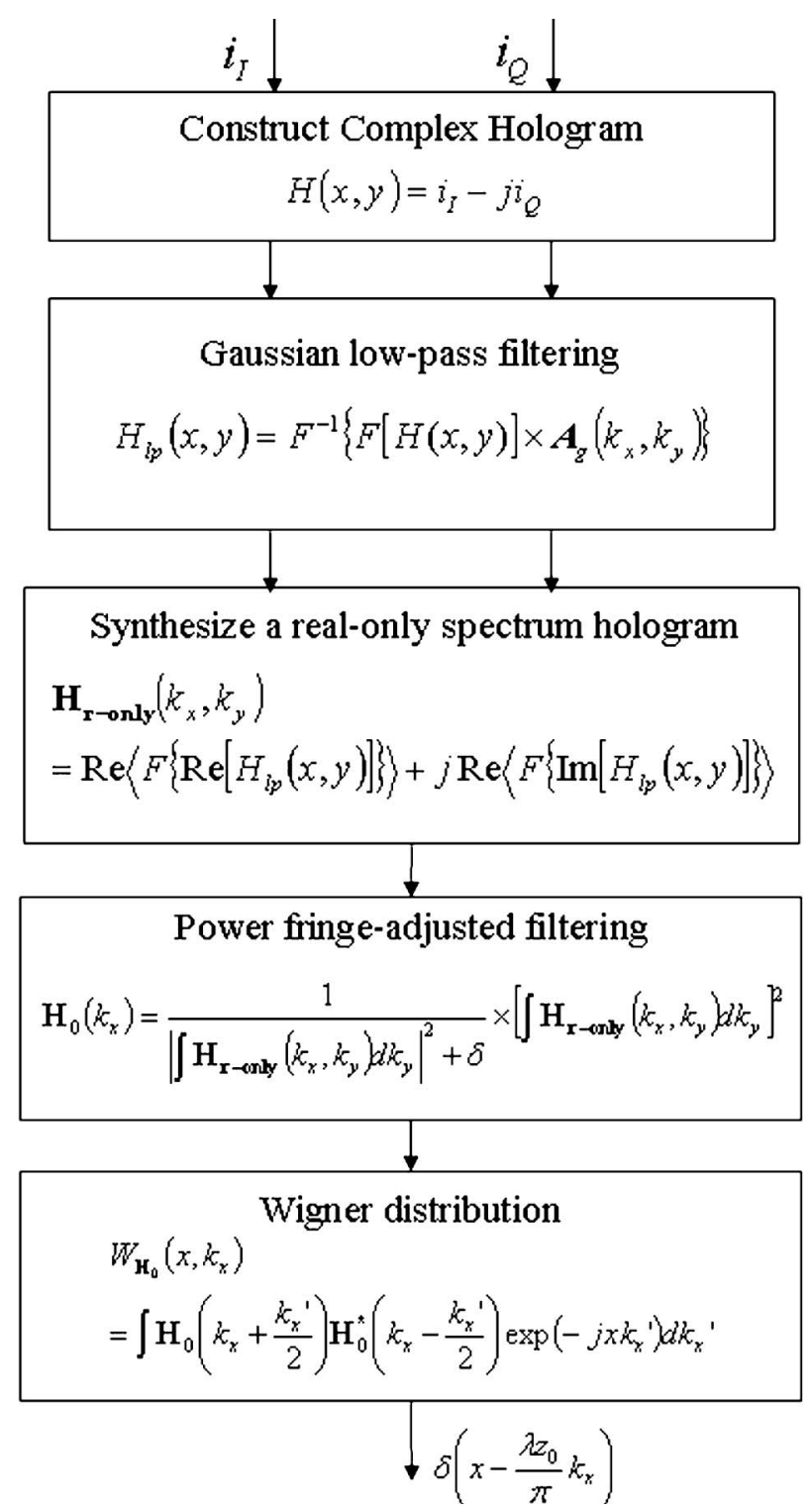

Fig. 2. Flow chart for extracting distance parameter $z_{0}$ from the hologram recorded by $\mathrm{OSH}$.

information only about distance parameter $z_{0}$. The phase term is extracted by the following process. First, the real-only spectrum hologram is projected in the $k_{y}$ direction. Second, the square of the projected real-only spectrum hologram is filtered by a power-fringe-adjusted filter [16], whose output is given by

$$
\begin{aligned}
\mathbf{H}_{0}\left(k_{x}\right) & =\frac{1}{\left|\int \mathbf{H}_{\mathbf{r}-\text { only }}\left(k_{x}, k_{y}\right) \mathrm{d} k_{y}\right|^{2}+\varepsilon}\left[\int \mathbf{H}_{\mathbf{r}-\text { only }}\left(k_{x}, k_{y}\right) \mathrm{d} k_{y}\right]^{2} \\
& \approx \exp \left(j \frac{\lambda z_{0}}{2 \pi} k_{x}^{2}\right),
\end{aligned}
$$

where $\varepsilon$ is a small value of either a constant or some function of $\left(k_{x}, k_{y}\right) ; \varepsilon$ is added to overcome the possible pole problems of the filter. Note that the output of the power-fringe-adjusted filter is the chirp signal along the $k_{x}$. The Wigner distribution of the chirp signal gives the line impulse with a slope of $d k_{x} / d x=$ $\pi / \lambda z_{0}$ on the frequency-space map $\left(x-k_{x}\right.$ plane). That is,

$$
\begin{aligned}
W_{\mathbf{H}_{\mathbf{0}}}\left(k_{x}, x\right)= & \int \mathbf{H}_{\mathbf{o}}\left(k_{x}+\frac{k_{x}{ }^{\prime}}{2}\right) \mathbf{H}_{\mathbf{0}}^{*}\left(k_{x}-\frac{k_{x}{ }^{\prime}}{2}\right) \\
& \times \exp \left(-j x k_{x}{ }^{\prime}\right) \mathrm{d} k_{x}{ }^{\prime} \propto \delta\left(x-\frac{\lambda z_{o}}{\pi} k_{x}\right) .
\end{aligned}
$$

Since the wavelength is known, we find distance parameter $z_{0}$ by measuring the slope of the line impulse. The last three blocks of the flow chart shown in Fig. 2 summarize the procedures. We have just now extracted the distance parameter from the hologram recorded by OSH. In Section 3 we describe the reconstruction of the image of the object using the distance parameter.

\section{Reconstruction with a Distance Parameter}

The reconstruction of the object from the complex hologram is achieved by wave propagation, which corresponds physically to optical reconstruction of the hologram by illuminating the hologram with a plane reconstruction beam. The image at a depth location of $z_{r}$ is reconstructed by convolution between the complex hologram and the complex conjugate of the FZP at the matched depth location, which is given by

$$
\begin{aligned}
I_{r}\left(x, y, z_{r}\right) & =H(x, y) \otimes h_{z_{r}}^{*}(x, y) \\
& =I_{0}\left(x, y, z_{r}\right)+\int_{\substack{z_{0}+(1 / 2) \delta \mathrm{z} \\
z_{0}-(1 / 2) \delta \mathrm{z} \\
z \neq z_{r}}} I_{0}(x, y, z) \\
& \otimes \frac{j A_{s}(x, y ; z)}{\lambda\left(z-z_{r}\right)} \exp \left[-j \frac{\pi}{\lambda\left(z-z_{r}\right)}\left(x^{2}+y^{2}\right)\right] \mathrm{d} z .
\end{aligned}
$$

Here we can see that the reconstructed image consists of the focused image at depth location $z_{r}$ [the first term in Eq. (11)] and the defocused noise [the second term in Eq. (11)]. Since the object is distributed along the depth axis from $z_{0}-(1 / 2) \delta z$ to $z_{0}+(1 / 2) \delta z$, i.e., the depth range of the object, reconstruction with $z_{r}$ within the depth range of the object yields a full 3-D image.

\section{Experimental Results}

We record the hologram of a specimen using OSH (see Fig. 1). As shown in Fig. 3, the specimen or the 3 -D object consists of two slides. The front and back slides are transparencies of a triangle and a rectangle, respectively. The lateral size of both slides is approximately $1 \mathrm{~cm} \times 1 \mathrm{~cm}$, and the depth distance between the two slides is $15 \mathrm{~cm}$. The $\mathrm{He}-\mathrm{Ne}$ laser with wavelength $\lambda=633 \mathrm{~nm}$ is used to generate coherent light. The diameter of the beam expanded by beam expanders BE1 and BE2 is $D=15 \mathrm{~mm}$ 
and the expanded beam from $\mathrm{BE} 1$ is focused by lens $\mathrm{L} 1$ with a focal length of $f=400 \mathrm{~mm}$. Thus, the NA of the scanning beam is $\mathrm{NA}=\sin [D /(2 f)]=0.01875$ and the Rayleigh range of the scanning beam is $z_{R} \approx$ $2 \lambda / \pi \mathrm{NA}^{2}=1.15 \mathrm{~mm}$. We positioned the object $87 \mathrm{~cm}$ away from the focal point of the spherical wave and scanned the 3-D object. The complex hologram of the object is recorded by adding the two outputs of OSH as in Eq. (2). Figures 4(a) and 4(b) show the amplitude and phase of the complex hologram. To extract the distance parameter, we first filter the hologram using a Gaussian low-pass filter with $\mathrm{NA}_{g}=$ 0.00116 , resulting in

$$
\Delta z=2 \lambda / \pi \times\left(\mathrm{NA}^{2}+\mathrm{NA}_{g}^{2}\right) /\left(\mathrm{NA}_{g} \mathrm{NA}\right)^{2} \approx 30 \mathrm{~cm},
$$

which is two times larger than the depth range of the object and is required to obtain the result in Eq. (7). Afterward, we synthesized the real-only spectrum hologram as given by Eq. (8). Finally, we revealed distance parameter $z_{0}$ as a delta line on a spacefrequency map using the Wigner distribution after power-fringe-adjusted filtering. Figure 5 shows the delta line on the space-frequency map, and the slope of the delta line is measured to be $\pi / \lambda z_{0} \approx$ $5.7 \mathrm{rad} / \mathrm{mm}^{2}$. According to the slope of the delta line, the distance parameter of the hologram is calculated to be $z_{0} \approx 87 \mathrm{~cm}$. Using the distance parameter, we reconstructed the image at the depth location of the object according to Eq. (11). The reconstructed image with $z_{r} \approx 87 \mathrm{~cm}$ is shown in Fig. 6 . The subsequent focusing within the depth range of the object, i.e., $z_{0}-(1 / 2) \delta z \leq z_{r} \leq z_{0}+(1 / 2) \delta z$, is performed by reconstruction according to Eq. (11), and the 3-D image of the reconstructed image of the object is shown in Fig. 7. Note that the triangle and rectangle are focused sharply at the front and the back of the reconstructed 3-D image, respectively.

\section{Concluding Remarks}

We have shown that autofocusing in OSH is possible without any searching algorithm or tracking process. The idea is to extract the distance parameter from the complex hologram. The complex hologram of an

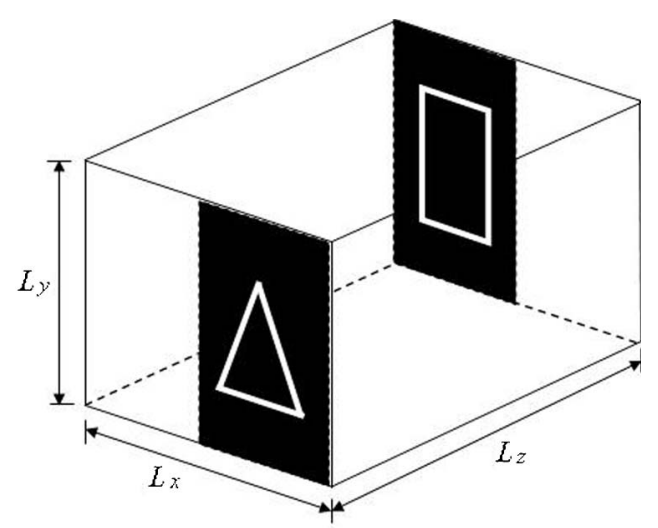

Fig. 3. Object with $L_{x}=L_{y}=1.5 \mathrm{~cm}$ and $L_{z}=15 \mathrm{~cm}$.
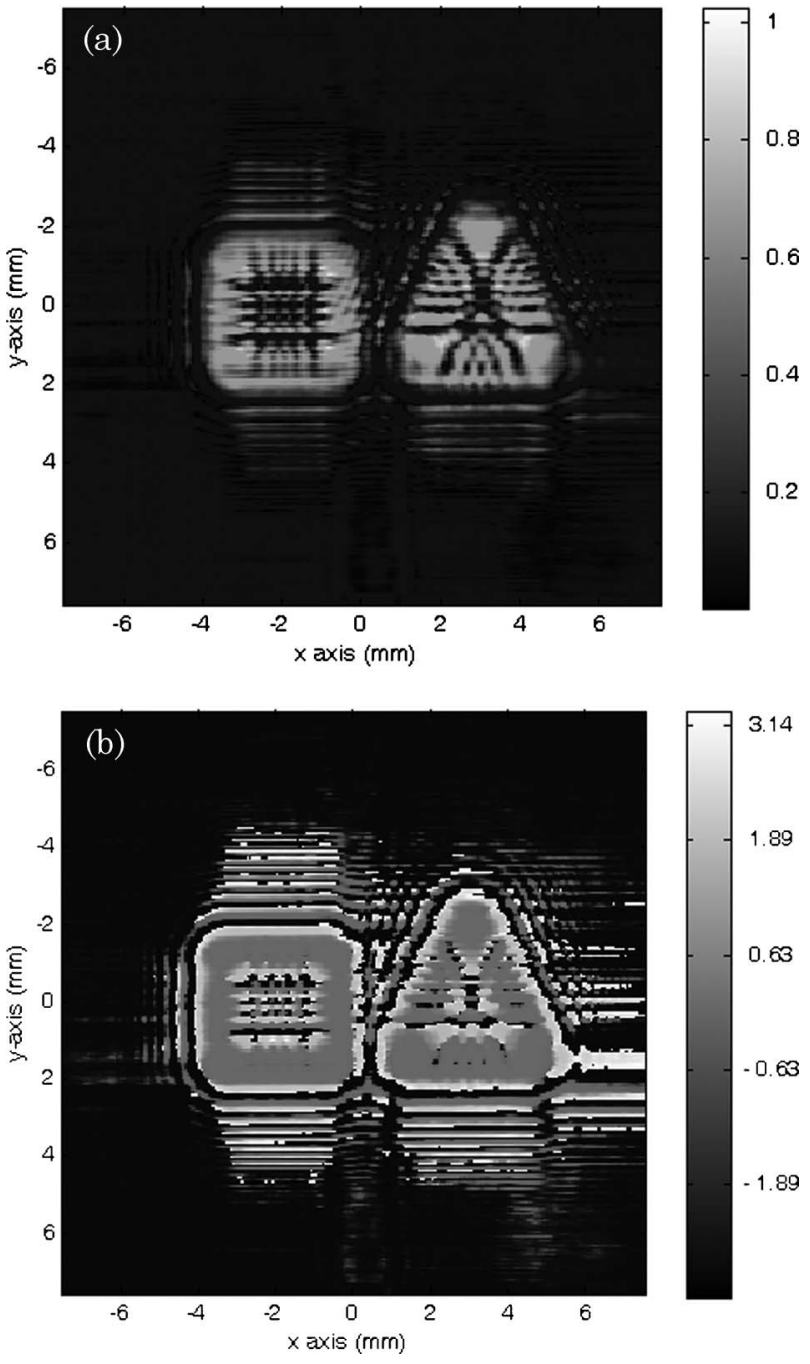

Fig. 4. (a) Amplitude and (b) phase of the complex hologram.

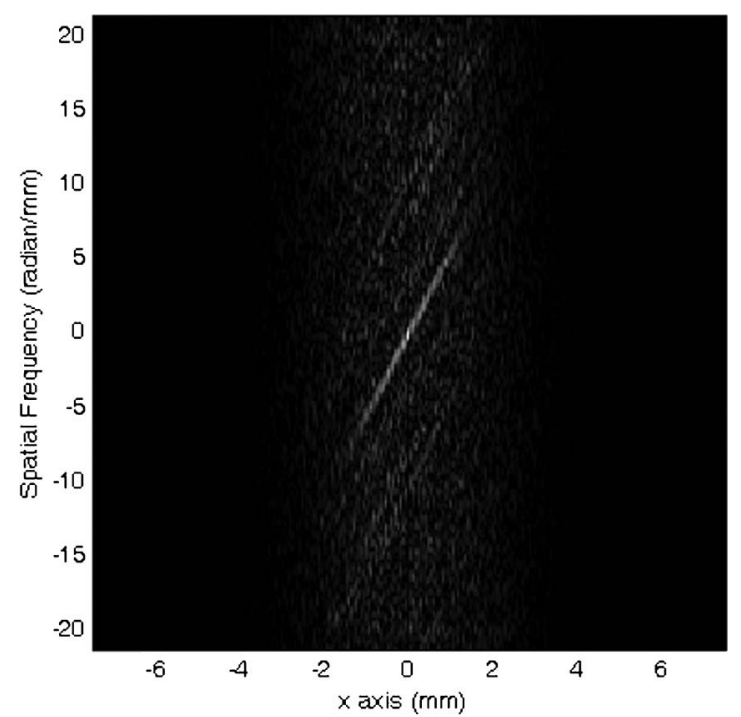

Fig. 5. Line impulse on the frequency-space map. 


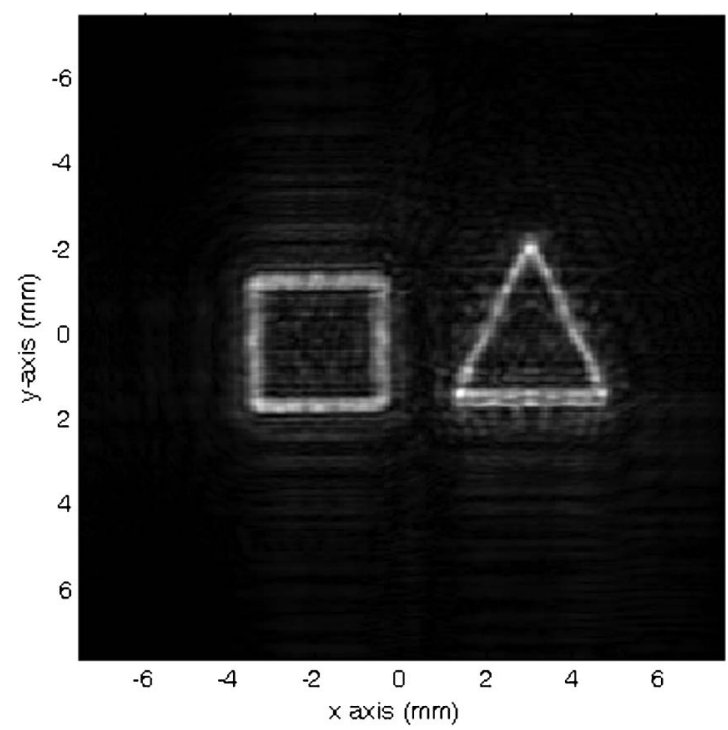

Fig. 6. Reconstructed image using distance parameter $z_{0}$.

intensity object is recorded holographically using the incoherent mode of OSH, and we set the FZP that codes the object to be constant within the depth range of the object by Gaussian low-pass filtering. Afterward, the real-only spectrum hologram is synthesized. Since the phase of the real-only spectrum hologram contains information about the distance parameter, we extract the phase term of the real-only spectrum hologram using power-fringe-adjusted filtering. The Wigner distribution of the power-fringeadjusted filtered real-only spectrum hologram reveals the distance parameter of the hologram as a delta line on a space-frequency map. We subsequently reconstructed the 3-D image of the object by focusing the images obtained from the recorded hologram using the distance parameter. To the best of our knowledge, this is the first time that autofocusing has been shown to be possible without any searching algorithm or tracking process. The proposed algorithm is for holograms of incoherent objects and, therefore, is also applicable to holograms obtained by space-time digital holography, Fresnel

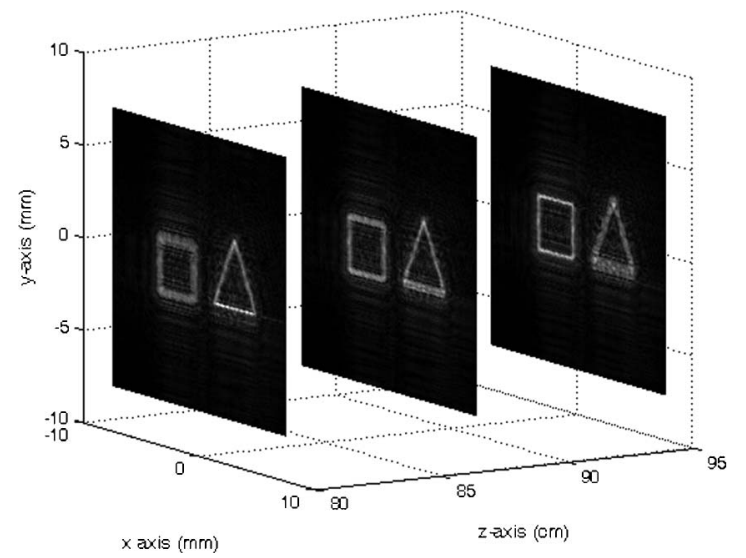

Fig. 7. Sequential reconstruction of the complex hologram shown in Fig. $\underline{4}$. incoherent correlation holography, computergenerated holography, and integral holography [17-21]. The proposed algorithm, however, is not applicable for coherent objects such as phase objects in biological cells. As an explanation, we note that, to derive Eq. (9) from Eqs. (7) and (8), for example, as one of the terms in Eq. (8), we have

$$
\begin{aligned}
\operatorname{Re}\left[H_{l p}(x, y)\right]= & \operatorname{Re}\left[\int_{z_{0}-(1 / 2) \delta z}^{z_{0}+(1 / 2) \delta z} I_{0}(x, y, z) \mathrm{d} z\right. \\
& \left.\otimes \frac{j A_{l p}\left(x, y ; z_{0}\right)}{\lambda z_{0}} \exp \left[-j \frac{\pi}{\lambda z_{0}}\left(x^{2}+y^{2}\right)\right]\right] \\
= & \int_{z_{0}+(1 / 2) \delta z}^{z_{0}-(1 / 2) \delta z} I_{0}(x, y, z) \mathrm{d} z \\
& \otimes \operatorname{Re}\left[\frac{j A_{l p}\left(x, y ; z_{0}\right)}{\lambda z_{0}} \exp \left[-j \frac{\pi}{\lambda z_{0}}\left(x^{2}+y^{2}\right)\right]\right] \\
& z_{0}+(1 / 2) \delta z \\
& \int_{z_{0}-(1 / 2) \delta z}^{I_{0}(x, y, z) \mathrm{d} z} \\
& \otimes \frac{A_{l p}\left(x, y ; z_{0}\right)}{\lambda z_{0}} \sin \left[\frac{\pi}{\lambda z_{0}}\left(x^{2}+y^{2}\right)\right],
\end{aligned}
$$

which is possible since $I_{0}(x, y, z)$ is an intensity object. However, if the object under consideration is coherent so that $I_{0}(x, y, z)$ is now replaced by

$$
O(x, y, z)=|O(x, y, z)| \exp (j \angle O)=\operatorname{Re}[O]+j \operatorname{Im}[O],
$$

where $\angle O$ is the phase angle of $O(x, y, z)$, Eq. $(\underline{12})$ becomes

$$
\begin{aligned}
\operatorname{Re}\left[H_{l p}(x, y)\right]= & \operatorname{Re}\left[\int_{z_{0}-(1 / 2) \delta z}^{z_{0}+(1 / 2) \delta z} O(x, y, z) \mathrm{d} z\right. \\
& \left.\otimes \frac{j A_{l p}\left(x, y ; z_{0}\right)}{\lambda z_{0}} \exp \left[-j \frac{\pi}{\lambda z_{0}}\left(x^{2}+y^{2}\right)\right]\right] \\
= & \int_{z_{0}-(1 / 2) \delta z}^{z_{0}+(1 / 2) \delta z} \operatorname{Re}[O(x, y, z)] \mathrm{d} z \\
& \otimes \frac{A_{l p}\left(x, y ; z_{0}\right)}{\lambda z_{0}} \sin \left[\frac{\pi}{\lambda z_{0}}\left(x^{2}+y^{2}\right)\right] \\
& -\int_{z_{0}+(1 / 2) \delta z} \operatorname{Im}[O(x, y, z)] \mathrm{d} z \\
& \otimes \frac{z_{l p}-(1 / 2) \delta z}{\lambda z_{0}} \cos \left[\frac{\pi}{\lambda z_{0}}\left(x^{2}+y^{2}\right)\right] .
\end{aligned}
$$

In Eq. (13) we see that there is an extra term, $\operatorname{Im}[O(x, y, z)]$, mixed with the cosine term, and because of it we could not synthesize the real-only 
spectrum hologram in Eq. (9). It is interesting to find some ways to overcome this problem of mixing for coherent objects, which we plan to investigate in the near future. The positive result of such an investigation would allow us to apply autofocusing for OSH working in the coherent mode [4] as well as for traditional holograms.

This research was supported by the Basic Science Research Program through the National Research Foundation of Korea (NRF) funded by the Ministry of Education, Science, and Technology, 20090087476. The authors also thank the reviewers for their helpful comments and suggestions.

\section{References}

1. T.-C. Poon, "Scanning holography and two-dimensional image processing by acousto-optic two-pupil synthesis," J. Opt. Soc. Am. A 2, 521-527 (1985).

2. T.-C. Poon, K. B. Doh, B. W. Schilling, M. H. Wu, K. K. Shinoda, and Y. Suzuki, "Three-dimensional microscopy by optical scanning holography," Opt. Eng. 34, 1338-1344 (1995).

3. B. W. Schilling, T.-C. Poon, G. Indebetouw, B. Storrie, K. Shinoda, Y. Suzuki, and M. H. Wu, "Three-dimensional holographic fluorescence microscopy," Opt. Lett. 22, 1506-1508 (1997).

4. G. Indebetouw, P. Klysubun, T. Kim, and T.-C. Poon, "Imaging properties of scanning holographic microscopy," J. Opt. Soc. Am. A 17, 380-390 (2000).

5. G. Indebetouw and W. Zhong, "Scanning holographic microscopy of three-dimensional fluorescent specimens," J. Opt. Soc. Am. A 23, 1699-1707 (2006).

6. G. Indebetouw, Y. Tada, J. Rosen, and G. Brooker, "Scanning holographic microscopy with resolution exceeding the Rayleigh limit of the objective by superposition of off-axis holograms," Appl. Opt. 46, 993-1000 (2007).

7. T.-C. Poon, Digital Holography and Three-Dimensional Display (Springer, 2006).

8. P. Ferraro, G. Coppola, S. De Nicola, A. Finizio, and G. Pierattini, "Digital holographic microscope with automatic focus tracking by detecting sample displacement in real time," Opt. Lett. 28, 1257-1259 (2003).

9. M. Liebling and M. Unser, "Autofocus for digital Fresnel holograms by use of a Fresnelet-sparsity criterion," J. Opt. Soc. Am. A 21, 2424-2430 (2004).

10. P. Langehanenberg, B. Kemper, D. Dirksen, and G. von Bally, "Autofocusing in digital holographic phase contrast microscopy on pure phase objects for live cell imaging," Appl. Opt. 47, D176 (2008).

11. T. Kim, Y. S. Kim, W. S. Kim, and T.-C. Poon, "Algorithm for converting full-parallax holograms to horizontalparallax-only holograms," Opt. Lett. 34, 1231-1233 (2009).

12. T. Kim and T.-C. Poon, "Extraction of 3-D location of matched 3-D object using power fringe-adjusted filtering and Wigner analysis," Opt. Eng. 38, 2176-2183 (1999).

13. T. Kim, T.-C. Poon, and G. Indebetouw, "Depth detection and image recovery in remote sensing by optical scanning holography," Opt. Eng. 41, 1331-1338 (2002).

14. T.-C. Poon, T. Kim, G. Indebetouw, B. W. Schilling, M. H. Wu, K. Shinoda, and Y. Suzuki, "Twin-image elimination experiments for three-dimensional Images in optical scanning holography," Opt Lett. 25, 215-217 (2000).

15. T.-C. Poon, "Three-dimensional television using optical scanning holography," J. Inf. Disp. 3, 12-16 (2002).

16. M. S. Alam, X.-W. Chen, and M. A. Karim, "Distortioninvariant fringe-adjusted joint transform correlation," Appl. Opt. 36, 7422-7427 (1997).

17. G. Indebetouw and P. Klysubun, "Space-time digital holography: a three-dimensional microscopic imaging scheme with an arbitrary degree of spatial coherence," Appl. Phys. Lett. 75, 2017-2019 (1999).

18. J. Rosen and G. Brooker, "Non-scanning motionless fluorescence three-dimensional holographic microscopy," Nat. Photon. 2, 190-195 (2008).

19. T.-C. Poon, "Holography: scan-free three-dimensional imaging," Nat. Photon. 2, 131-132 (2008).

20. Y. Li, D. Abookasis, and J. Rosen, "Computer-generated holograms of three-dimensional realistic objects recorded without wave interference," Appl. Opt. 40, 2864-2870 (2001).

21. N. T. Shaked, J. Rosen, and A. Stern, "Integral holography: white-light single-shot hologram acquisition," Opt. Express 15, 5754-5760 (2007). 